\title{
Primary mesenchymal chondrosarcoma with bilateral kidney invasion and calcification in renal pelvis: A case report and review of the literature
}

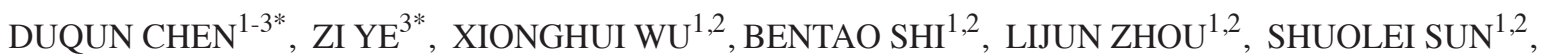 \\ BENLIN WEI $^{1,2}$, SHANGQI YANG ${ }^{1,2}$, XIANGMING MAO $^{1,2}$ and YONGQING LAI ${ }^{1,2}$ \\ ${ }^{1}$ Department of Urology, Peking University Shenzhen Hospital, Shenzhen, Guangdong 518036; \\ ${ }^{2}$ Guangdong and Shenzhen Key Laboratory of Male Reproductive Medicine and Genetics, Institute of Urology, \\ Peking University Shenzhen Hospital, Shenzhen PKU-HKUST Medical Center, Shenzhen, Guangdong 518036; \\ ${ }^{3}$ Department of Infectious Disease, Anhui Medical University First Affiliated Hospital, Hefei, Anhui 230032, P.R. China
}

Received July 31, 2014; Accepted April 9, 2015

DOI: $10.3892 / \mathrm{ol} .2015 .3252$

\begin{abstract}
Mesenchymal chondrosarcoma (MC) is a rare malignant cartilaginous forming tumor. MC of the kidney is extremely rare, with only seven cases reported in the literature. The present study described the case of a 17-year-old male, who presented with sudden severe pain in the right flank and a high fever. Imaging studies demonstrated a large soft heterogeneous mass $(7.8 \times 9.5 \times 15 \mathrm{~cm})$ located between the liver and right kidney with no clear demarcation, and a well-demarcated mass $(1.3 \times 2.4 \mathrm{~cm})$ with patchy dense calcification occupying the left renal pelvis. Following the diagnosis of a Wilms' tumor, the patient underwent a right radical nephrectomy and the pathological diagnosis was MC of the kidney. To the best of our knowledge, the current study presents the first case of MC with bilateral kidney invasion and calcification in the renal pelvis. In addition, the clinical, radiological and pathological features, and the management of this unusual neoplasm were discussed.
\end{abstract}

\section{Introduction}

Mesenchymal chondrosarcoma (MC) is a rare neoplasm, constituting $\sim 1 \%$ of all chondrosarcomas (1). Almost $24 \%$ of cases of this rare tumor originate from an extraskeletal site, and the predominant extraosseous site is the head and neck

Correspondence to: Professor Yongqing Lai or Professor Xiangming Mao, Department of Urology, Peking University Shenzhen Hospital, 1120 Lianhua Road, Shenzhen, Guangdong 518036, P.R. China

E-mail: yqlord@163.com

E-mail: 13691903635@126.com

*Contributed equally

Key words: mesenchymal chondrosarcoma, bilateral kidney invasion, calcification, renal pelvis region, followed by the extremities and trunk (2). MC cases that arise in the kidney are extremely rare and, to the best of our knowledge, only seven cases have previously been reported in the literature (2-8). Wilms' tumor is the most common type of renal malignancy in children. Classically, a radical nephrectomy for Wilms' tumor has been recommended as the standard option (9).

The present study describes the eighth case of primary renal MC, initially misdiagnosed as Wilm's tumor, which presented with a large right renal mass with hemorrhage and a second mass with calcification in the left renal pelvis. To the best of our knowledge, the present case is the first MC with bilateral kidney invasion and calcification in the renal pelvis.

\section{Case report}

A 17-year-old male presented with sudden severe pain in the right flank and high fever, and was admitted to the Shenzhen People's Hospital (Shenzhen, China) in May 2014. Laboratory tests were performed and the results were as follows: White

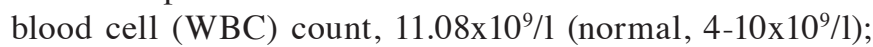
neutrophilic granulocyte percentage (N\%), 80.1\% (normal, $50-70 \%$ ); red blood cell (RBC) count, 5.64x10 12/1 (normal, 4.0-5.5 $\times 10^{12} / 1$ ); hemoglobin (HBG) level, $156 \mathrm{~g} / 1$ (normal, 120-160 g/l); aspartate aminotransferase (AST) level, $243 \mathrm{U} / 1$ (normal, 0-40 U/1); and alanine aminotransferase (ALT) level, $201 \mathrm{U} / 1$ (normal, 0-40 U/1). Imaging studies using ultrasonography and abdominal computerized tomography $(\mathrm{CT})$ indicated a right $10-\mathrm{cm}$ heterogeneous renal mass and calcification in the left renal pelvis. The patient was then diagnosed with a right renal tumor with hemorrhage and a left renal pelvis calculus, and transferred to the Peking University Shenzhen Hospital (Shenzhen, China) for further treatment. Physical examination demonstrated percussion pain over the right kidney region. Laboratory tests were performed and demonstrated the following levels: WBC count, $13.08 \times 10^{9} / 1$; N\%, 82.3\%; RBC count, 5.06 $\times 10^{12} / 1$; and HBG level, $145 \mathrm{~g} / \mathrm{l}$. Tumor markers, such as $\alpha$-fetoprotein, urinalysis and kidney function were within the normal range. Enhanced CT scans demonstrated a 
A

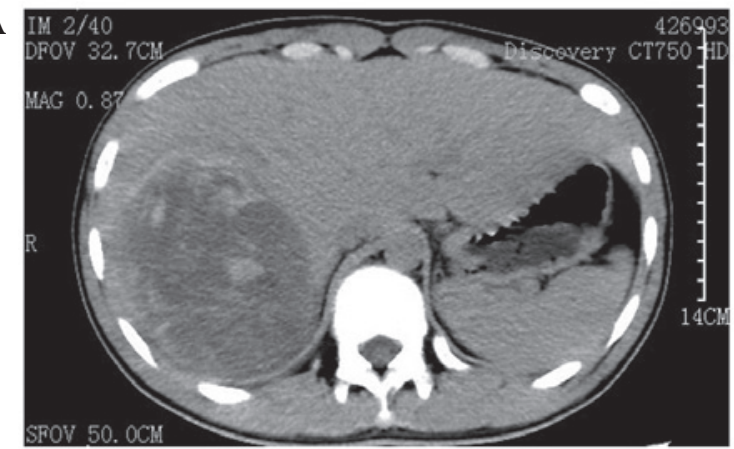

C

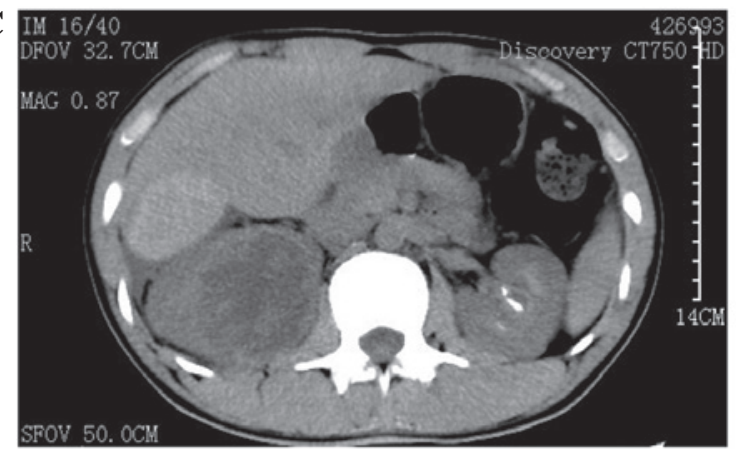

B

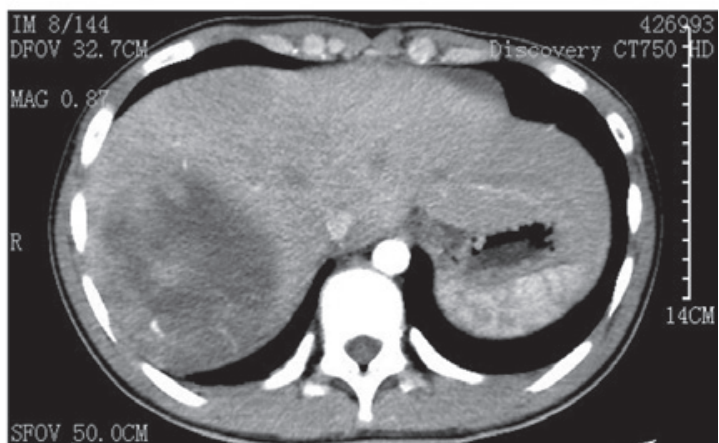

D

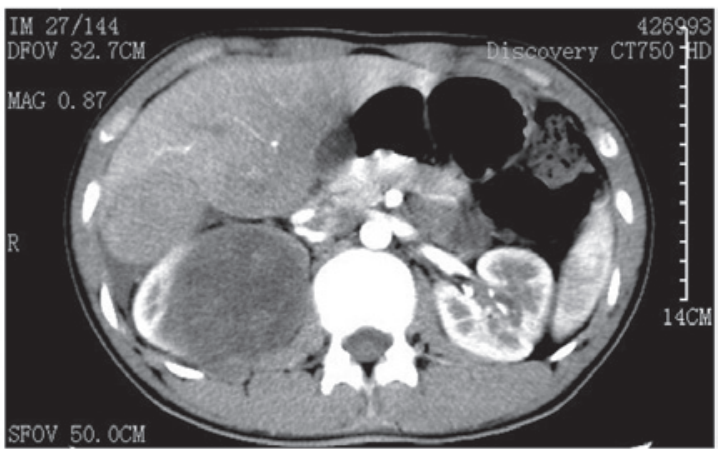

Figure 1. (A) CT scan indicated a large heterogeneous mass in the upper pole of the right kidney and no clear demarcation with the liver, (B) with no enhancement. (C) Small soft-mass with patchy dense calcification occupied the renal pelvis, (D) with no enhancement.
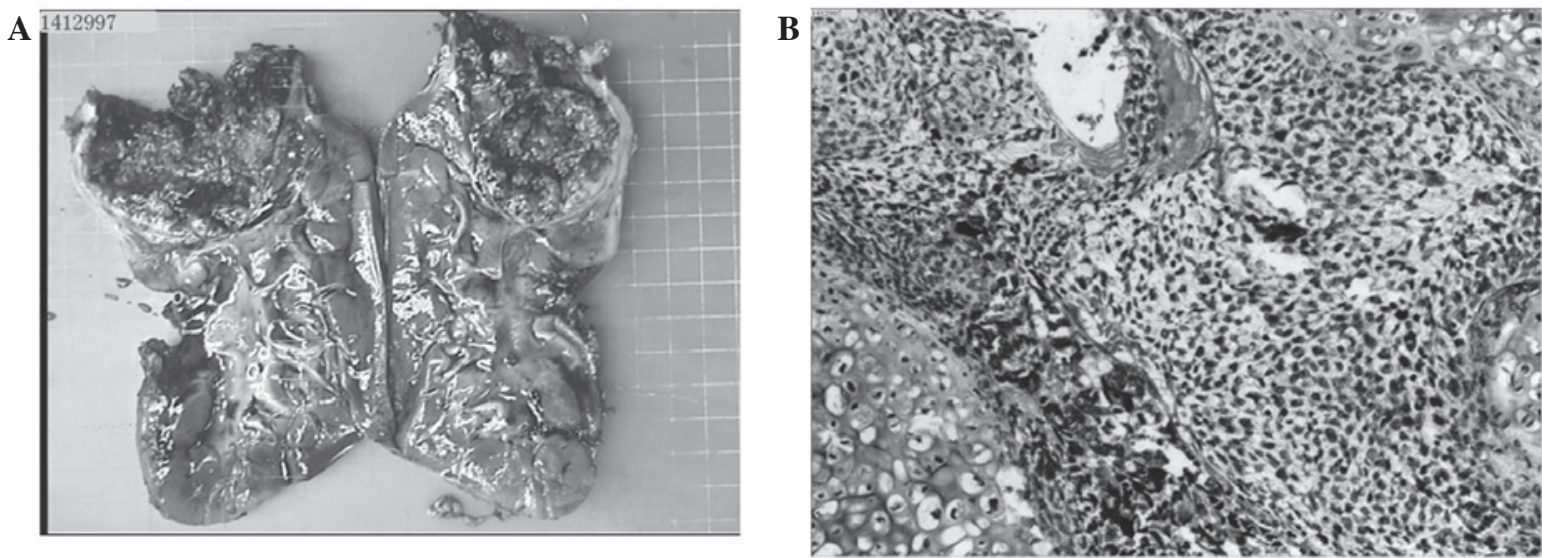

Figure 2. (A) Tumor with hemorrhage and necrosis located in the upper pole with no invasion into the renal pelvis. (B) Pathological examination demonstrated the islands of well-differentiated cartilage surrounded by sheets of small, spindle-shaped and undifferentiated tumor cells (hematoxylin and eosin staining; magnification, x200).

large soft heterogeneous mass $(7.8 \times 9.5 \times 15 \mathrm{~cm})$ located between the liver and right kidney with no clear demarcation (Fig. 1A). The CT number was 25-64 Hu and no enhancement was observed at the artery phase (Fig. 1B). The shape and size of the left kidney were normal; however, a well-demarcated mass $(1.3 \times 2.4 \mathrm{~cm})$ with patchy dense calcification occupied the renal pelvis (Fig. 1C), with no enhancement (Fig. 1D). No evidence of metastasis was observed on the CT scan. Consultation with the Department of Hepatobiliary Surgery (Peking University Shenzhen Hospital) excluded the possibility of liver metastasis and the patient's liver dysfunction was considered to be a result of tumor oppression. Liver supportive and anti-infective therapies were performed, and AST and ALT levels dropped to within the normal limits. However, the symptoms of high fever persisted $\left(39^{\circ} \mathrm{C}\right)$, which was interpreted as a result of the tumor hemorrhage, subsequent to excluding the possibility of infectious disease by a specialized infectious disease doctor.

Following an initial diagnosis of a Wilms' tumor, a right radical nephrectomy was performed. However, complications occurred during the surgery and the integrity of the tumor was destroyed. The tumor was a soft-tissue mass, which involved the upper pole of the right kidney and bulged into the perirenal fat. It had a grayish, dull-red appearance, was well-circumscribed and contained areas of hemorrhage and necrosis (Fig. 2A). Histological examination indicated a bimorphic growth pattern, which consisted of a sheath of undifferentiated spindle-shaped cells surrounded with islands of well-differentiated cartilage (Fig. 2B). Immunohistochemical analysis 
was performed and the tumor was found to be positive for cluster of differentiation (CD) 68, CD57, CD99 and myogenin, whereas it was negative for Wilms tumor protein 1, smooth muscle actin, epithelial membrane antigen and neuron-specific enolase, supporting the diagnosis of MC. The patient recovered from surgery without any postoperative complications. Bone scans and chest radiographs for disease staging were negative. During the 3 months of follow-up, the left renal tumor did not demonstrate any enlargement and no metastasis was observed. The patient underwent 6 cycles of chemotherapy consisting of doxorubicin $\left(70 \mathrm{mg} / \mathrm{m}^{2}\right.$, day 1) and cyclophosphamide (700 mg/m², day 1), each cycle lasted 28 days. The left renal tumor did not demonstrate any enlargement and no metastasis was observed 10 months after surgery.

Written informed consent for the present study was obtained from the patient.

\section{Discussion}

$\mathrm{MC}$ is a rare, high-grade malignancy of the bone or soft tissue, with a unique, biphasic histology and poor prognosis. Due to its rarity and variable length of disease-free survival rates, the disease course of MC remains poorly understood (10). MC arising in the brain and the meninges, retroperitoneum and the extremities has previously been reported, but $\mathrm{MC}$ arising in the kidney is extremely rare (10). A review of the literature was conducted through searching the PubMed database (http:// www.ncbi.nlm.nih.gov/pubmed) for English language papers published between its inception and 28th July 2014, using the terms 'renal' or 'kidney' and 'mesenchymal chondrosarcoma'; seven cases of primary renal MC were identified (Table I) (2-8). Men and women are equally affected by MC, most frequently in the second to fourth decade of life (average age, 25 years). Clinical symptoms are nonspecific and include pain, swelling, and a palpable soft tissue mass (11). As presented in Table I, three MC patients were male and four were female, and the age range was 22-64 years with a mean age of 45 years, which may be explained by the limited number of cases (2-8). To the best of our knowledge, the patient described in the present study is the youngest patient diagnosed with MC. As in the present case, the majority of the MC patients presented with flank pain and gross hematuria, incidental mass, macroscopic hematuria by examination and high fever. The MC tumors ranged in size between 2.5 and $12 \mathrm{~cm}$ with a mean size of $8 \mathrm{~cm}(2-8)$. At present, the left renal tumor in the patient of the current study is the smallest MC observed in the kidney. The majority of kidney MC tumors originated in the renal parenchymal (6/7 cases)(2-7), while the other case originated in the renal pelvis (8). The current study described the first case of MC that presented with bilateral kidney invasion, with one tumor in the renal parenchymal region and one in the contralateral renal pelvis.

Radiologically, MCs usually present as soft-tissue masses with dense and granular calcification (12), as was observed in 5/7 cases documented by imaging (2-6), in addition to the left renal mass observed in the patient in the present case report. Renal MCs may also present as heterogeneous and hypodense masses without calcification (7-8), such as the right renal tumor in the patient in the current study. To the best of our knowledge, the present case study describes the first case with

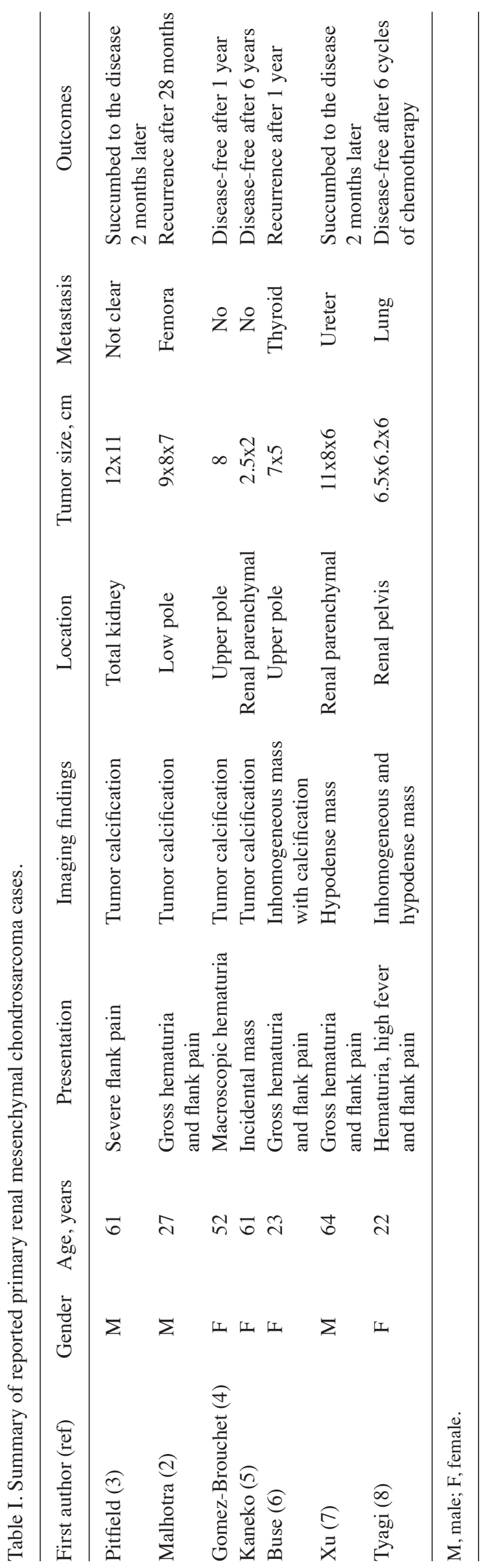


evidence of two MC kidney tumors identified on a CT scan. The diagnosis of primary MC is usually based on the typical histological appearance: Islands of well-differentiated cartilage surrounded by undifferentiated spindle mesenchymal cells (2). Further immunohistopathological analysis is also performed when possible. In addition, the possibility of metastasis of skeletal chondrosarcoma should be excluded by extensive bone imaging (bone scan, positron emission tomography-CT).

MCs are high-grade aggressive tumors. In the literature, 4/6 cases demonstrated metastasis at presentation; metastatic sites included the femora, thyroid, ureter and lung (2,6-8). The prognosis was poor, with two patients succumbing to the disease within 2 months after diagnosis $(3,7)$ and two patients experiencing recurrence $(2,6)$, which is similar to the estimated survival rate of $55 \%$ at 5 years and $27 \%$ at 10 years for extraskeletal MC (11). The recommended management for $\mathrm{MC}$ is with wide local resection and limb salvage when possible, and the use of neoadjuvant chemotherapy may be considered to control local disease and reduce the risk of metastasis (13). Kaneko et al (5) reported a patient with a tumor size of $2.5 \times 2 \mathrm{~cm}$, who underwent local excision and remained disease-free after 6 years, highlighting the importance of early diagnosis and treatment to achieve an improved prognosis.

In conclusion, $\mathrm{MC}$ is an extremely rare neoplasm in the kidney with poor prognosis. To the best of our knowledge, the present study described the first case of MC that presented with bilateral kidney invasion and calcification in the renal pelvis with a unique appearance on a CT scan. In addition, the clinical features were discussed, which may deepen the understanding of this unusual neoplasm.

\section{Acknowledgements}

This study was supported by grants from the National Natural Science Foundation of China (grant no. 81101922), the Medical Scientific Research Foundation of Guangdong Province of
China (grant nos. A2012584 and A2013606), the Science and Technology Development Fund Project of Shenzhen (grant no. JCYJ20130402114702124) and the fund of Guangdong Key medical subject.

\section{References}

1. Trembath DG, Dash R, Major NM and Dodd LG: Cytopathology of mesenchymal chondrosarcomas: A report and comparison of four patients. Cancer 99: 211-216, 2003.

2. Malhotra CM, Doolittle CH, Rodil JV and Vezeridis MP: Mesenchymal chondrosarcoma of the kidney. Cancer 54: 2495-2499, 1984.

3. Pitfield J, Preston BJ and Smith PG: A calcified renal mass: chondrosarcoma of kidney. Br J Radiol 54: 262, 1981.

4. Gomez-Brouchet A, Soulie M, Delisle MB and Escourrou G: Mesenchymal chondrosarcoma of the kidney. J Urol 166: 2305, 2001.

5. Kaneko T, Suzuki Y, Takata R, Takata K, Sakuma T and Fujioka T: Extraskeletal mesenchymal chondrosarcoma of the kidney. Int J Urol 13: 285-286, 2006.

6. Buse S, Behnisch W, Kulozik A, Autschbach F and Hohenfellner M: Primary chondrosarcoma of the kidney: Case report and review of the literature. Urol Int 83: 116-118, 2009.

7. Xu H, Shao M, Sun H and Li S: Primary mesenchymal chondrosarcoma of the kidney with synchronous implant and infiltrating urothelial carcinoma of the ureter. Diagn Pathol 7: 125, 2012.

8. Tyagi R, Kakkar N, Vasishta RK and Aggarwal MM: Mesenchymal chondrosarcoma of kidney. Indian J Urol 30: 225-227, 2014.

9. Gleason JM, Lorenzo AJ, Bowlin PR and Koyle MA: Innovations in the management of Wilms' tumor. Ther Adv Urol 6: $165-176,2014$

10. Shakked RJ, Geller DS, Gorlick R and Dorfman HD: Mesenchymal chondrosarcoma: Clinicopathologic study of 20 cases. Arch Pathol Lab Med 136: 61-75, 2012.

11. Murphey MD, Walker EA, Wilson AJ,Kransdorf MJ, Temple HT and Gannon FH: From the archives of the AFIP: Imaging of primary chondrosarcoma: radiologic-pathologic correlation. Radiographics 23: 1245-1278, 2003.

12. Hashimoto N, Ueda T, Joyama S, et al: Extraskeletal mesenchymal chondrosarcoma: An imaging review of ten new patients. Skeletal Radiol 34: 785-792, 2005.

13. Douis H and Saifuddin A: The imaging of cartilaginous bone tumours. II. Chondrosarcoma. Skeletal Radiol 42: 611-626, 2013. 